\title{
EMPIRICAL MODEL OF GALACTIC COSMIC RAY PARTICLE FLUXES BASED ON THE EXPERIMENTAL DATA IN SOLAR CYCLES 21-24
}

\author{
N. V. Kuznetsov, E. P. Popova, M. I. Panasyuk, M. V. Podzolko' \\ Skobeltsyn Institut of Nuclear Physics, Lomonosov Moscow State University \\ Leninskie gory 1(2), GSP-1, Moscow, 119991, Russian Federation \\ E-mail: spacerademail.ru
}

Fundamentals of a new model developed for predicting GCR particle fluxes during space missions are discussed. The model is based on the data set measured onboard spacecraft and stratospheric balloons from 1970s till 2015. The model describes fluxes of GCR particles with charge $z$ from 1 to 28 and energy from $\sim 80 \mathrm{MeV} /$ nucleon up to $100 \mathrm{GeV} /$ nucleon in the interplanetary space at heliocentric distance $\sim 1 \mathrm{AU}$ as a function of solar activity (averaged sunspot number).

35th International Cosmic Ray Conference - ICRC2017

10-20 July, 2017

Bexco, Busan, Korea

${ }^{1}$ Speaker 


\section{Introduction}

Fluxes of galactic cosmic ray (GCR) particles are essential components of cosmic radiation, affecting the spacecraft equipment and spaceship crews. At present time for predicting the GCR particle fluxes during the space missions several models are used, which computerbased versions are included in various software packages (including interactive). There are two widely used models, developed for the interplanetary space for heliocentric distance of $\sim 1 \mathrm{AU}$. NASA Badhwar \& O'Neill (BON) model $[1,2]$ is based on the solution of the equation of charged GCR particle transport from interstellar media into heliosphere, taking into account the variations of heliospheric environment due to the change of solar activity. Skobeltsyn Institute of Nuclear Physics (SINP) model [3] is purely empirical, summarizing the experimental data from monitors (detectors) and spectrometers from spacecraft and balloon experiments.

The main provisions of the mentioned models were formulated in 1980s and refined several times while receiving the new experimental data. Two versions of BON model (BON2004 and 2010) are included in OLTARIS software package (http://oltaris.larc.nasa.gov/). One of the first versions of SINP model is included into CREME96 software [4] (http://creme.isde.vanderbilt.edu/), and the last version of this model is approved as international standard [5].

In the last years for the engineering applications new empirical models [6, 7] were suggested, which using the common approach generalize the large amount of existing experimental data, including obtained in 23 and 24 cycles of solar activity.

In the current study the SINP-2016 model of GCR particle fluxes with energies from $\sim 80$ to $10^{5} \mathrm{MeV} /$ nucleon for interplanetary space at heliocentric distance of $\sim 1 \mathrm{AU}$ is discussed and the results of its applications are given.

\section{Energy spectra of proton and helium}

To obtain the analytic expression for the energy spectrum of particles we analyzed series of spectrometer data (BESS [8, 9], AMS [10, 11], PAMELA [12] etc.). It turned out that a good approximation of the experimental data (with an accuracy of $15 \%$ at $E>\sim 80 \mathrm{MeV} /$ nucleon) is observed if

$$
F_{(\mathrm{p}, \mathrm{He})}(E, t)=A_{(\mathrm{p}, \mathrm{He})} \cdot E^{-\gamma} \cdot\left(\frac{E}{E+\varepsilon_{(\mathrm{p}, \mathrm{He})}(t)}\right)^{\Delta},
$$

where $\gamma$ is power-index, characterizing the unmodulated particle spectrum at high energies $\left(E>20 \mathrm{GeV} /\right.$ nucleon); $\varepsilon_{(\mathrm{p}, \mathrm{He})}(t)$ is a parameter (modulation potential) depending on time $t$ and $\Delta=3.7$.

We applied $\gamma=2.72$ [13] for proton and helium and obtained a good approximation of the particle fluxes in the given energy interval (with mean deviation $\pm \sim 12 \%$ ) if $A_{(\mathfrak{p})}=1.7 \cdot 10^{5}$ $\left[(\mathrm{MeV} / \text { nucleon })^{1.72} /\left(\mathrm{cm}^{2} \cdot \mathrm{sr} \cdot \mathrm{s}\right)\right]$ for protons and $A_{(\mathrm{He})}=1.0 \cdot 10^{4}\left[(\mathrm{MeV} / \text { nucleon })^{1.72} /\left(\mathrm{cm}^{2} \cdot \mathrm{sr} \cdot \mathrm{s}\right)\right]$ for helium. 


\section{Energy spectrum of heavy charged particles}

Analyzing the CRIS/ACE data [14] in solar cycle 23 and 24 and the spectrometer data we concluded that the heavy charged particle (HCP) energy spectra multiplied by a constant coefficient (specific for different types of HCPs) coincided with a helium energy spectrum measured in the same time period. Figure 1 demonstrates the validity of this conclusion.

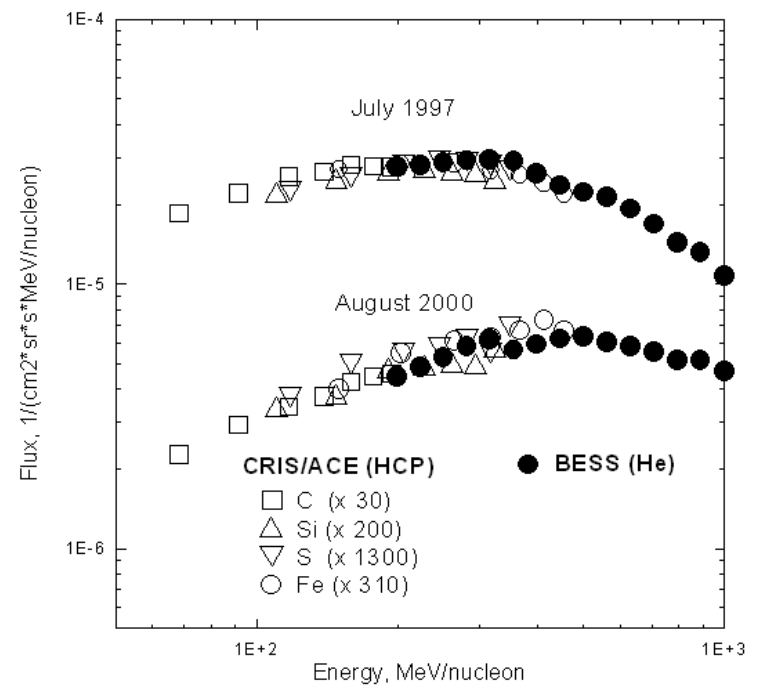

Figure 1. The experimental (CRIS/ACE) fluxes of some heavy nuclei (open badges) normalized to the experimental (BESS) fluxes of the helium (dark badges) at 1997 and 2000. The error of the data is limited by the size of the badges.

Therefore the HCP energy spectra are determined by the formula

$$
F_{(\mathrm{HCP})}(E, t)=\zeta_{(\mathrm{HCP})} F_{(\mathrm{He})}(E, t),
$$

where $\zeta_{(\mathrm{HCP})}$ is the normalization coefficient which we obtained (see [6]) from the experimental data (http://www.srl.caltech.edu/ACE/).

Thus, expressions (1) and (2) establish the GCR particle energy spectra $F(E, t)$ versus time $t$ if known the modulation potential $\varepsilon(t)$ versus time $t$.

\section{Modulation potential $\varepsilon(t)$}

To find the time dependence of modulation potential $\varepsilon(t)$ we substituted in formula (1) the experimental particle fluxes $F(E, t)$ measured by GME/IMP8 (protons and helium) $[15,16]$, EPHIN/SOHO (proton) [17] and CRIS/ACE (HCPs, http://www.srl.caltech.edu/ACE/) monitors. Figure 2 shows the temporal dependence of $\varepsilon_{\exp }(t)$ for protons and helium in comparison with the temporal dependence of the smoothed monthly mean sunspot numbers $W(t)$.

To improve the correlation between the $\varepsilon_{\exp }(t)$ and $W(t)$ a known effect $[18,19]$ of the time delays $W(t) \rightarrow W(t-\Delta t)$ in odd $\left(\Delta t_{\text {odd }}\right)$ and even $\left(\Delta t_{\text {even }}\right)$ solar cycles was taken into account. It turned out that the maximum value of the correlation coefficient between $\varepsilon(t)$ and $W(t-\Delta t)$ is at $\Delta t_{\text {odd }}=15.5$ and $\Delta t_{\text {even }}=5.5$ months. 


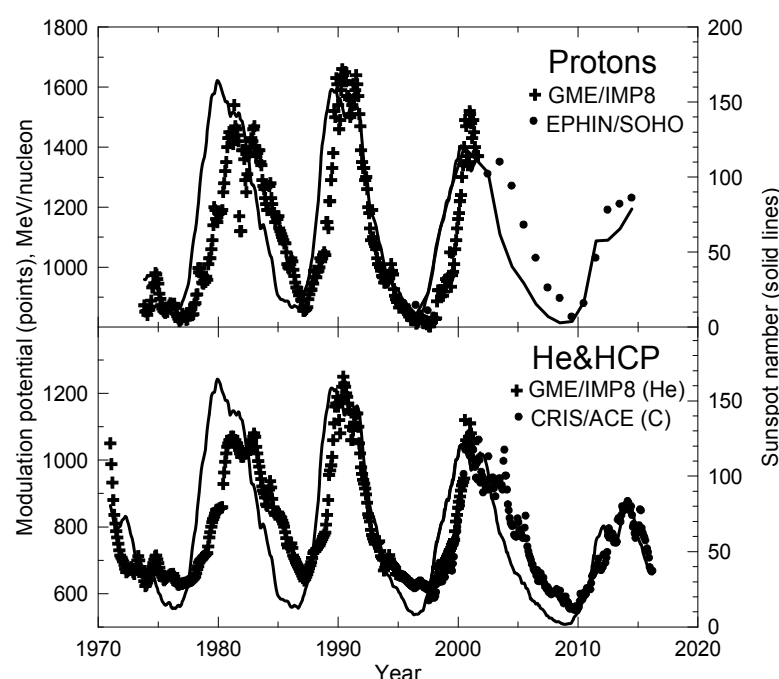

Figure 2. The experimental

temporal dependencies of modulation potential $\varepsilon_{\text {exp }}(t)$ (points) and International smoothed monthly mean sunspot numbers v.1 $W(t)$ (solid curves).

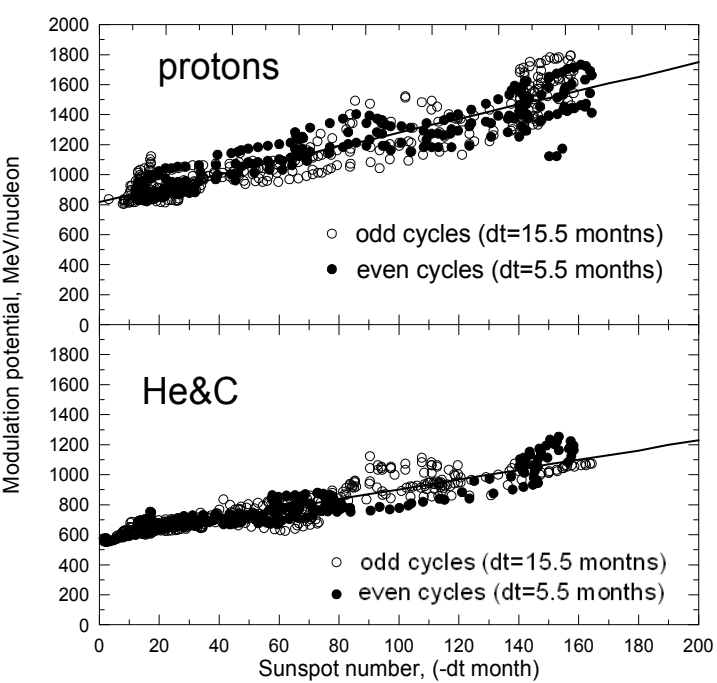

Figure 3. Scatter plot for protons (top panel) and helium \& carbon (bottom panel). Straight lines are linear dependency (3) with the average parameters $\varepsilon_{0}$ and $\kappa$ (see the text).

Using the adopted values $\Delta t_{\text {odd }}$ and $\Delta t_{\text {even }}$ the scatter plot $\varepsilon_{\text {exp }}(t) \& W(t-\Delta t)$ was constructed (Figure 3). As we see in Fig. 3 a linear relationship between $\varepsilon_{\text {exp }}(t) \& W(t-\Delta t)$ exists

$$
\varepsilon(t)=\varepsilon_{0}+\kappa \cdot W(t-\Delta t)
$$

To determine the mean values and standard deviations from the mean values for the $\varepsilon_{0}$ and $\kappa$ parameters in expression (3) the least squares method was used. The straight lines in Figure 3 are the dependencies (3) with the parameters: $\varepsilon_{0(\mathrm{p})}=817 \pm 8.5 \mathrm{MeV} /$ nucleon and $\kappa_{(\mathrm{p})}=4.64 \pm 0.08$ for protons and $\varepsilon_{0(\mathrm{He})}=576 \pm 4 \mathrm{MeV} /$ nucleon and $\kappa_{(\mathrm{He})}=3.26 \pm 0.05$ for helium.

\section{Comparison of model and experimental data}

In Figures 4 we can see the examples of comparison of typical temporal dependencies of GCR particle fluxes that was measured by the different monitors and calculated by the SINP2016 model.

In top panels of Figures 4 we can see the irregular fluctuations of deviation between the experimental and calculated data $\left(F_{\text {exp }}-F_{\text {cal }}\right) / F_{\text {cal }}$ that sometimes can reach $\pm \sim 50 \%$ in the phase of solar activity increase and near the solar maximum. We can associate these fluctuations with fluctuations that occur in the solar wind and the interplanetary magnetic field that the model cannot take into account. The mean deviation between calculated and experimental data for long-term intervals (about and more than solar cycle) is within the range of experimental errors about $\pm 10-15 \%$ which is within the usual range of experimental errors. 

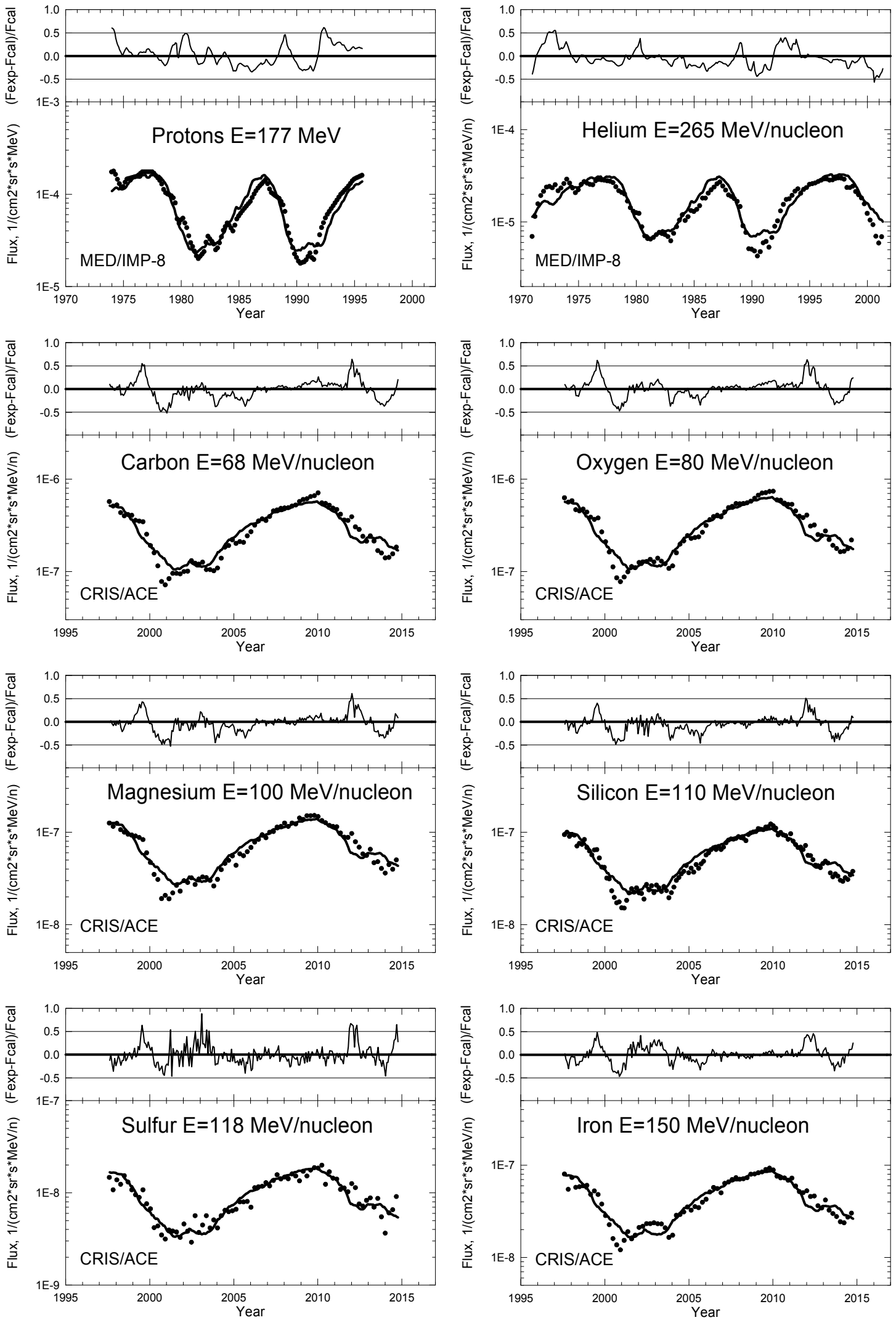

Figure 4. Temporal dependence of the experimental (points) and model (curves) GCR particle fluxes (the bottom panels) and deviations between experimental and calculated data (the top panels). 


\section{Conclusion}

In the current study the main provisions of SINP-2016 empirical model of GCR particle fluxes, developed for the engineering applications, are discussed. The analytical expressions and the parameters of the model generalize the data, obtained by monitors and spectrometers in 2124 solar activity cycles. The model establishes the relation between the sunspot number and the fluxes of GCR particles with $z=1-28$ ) in the interplanetary space in the ecliptic plane at heliocentric distance of $\sim 1$ AU. The accuracy of the model is estimated at the level of $\pm 50 \%$ during the phase of solar activity increase and near the solar maximum and not worse than $\pm 20 \%$ during the phase of solar activity decrease.

\section{Acknowledgments}

This work was funded by Russian Science Foundation by Grant 17-29-01022.

\section{References}

[1] G.D. Badhwar and P.M. O’Neill, Galactic cosmic radiation model, Adv. Space Res. 17 (1996) 207.

[2] P.M. O'Neill, S. Golge and T.C. Slaba, Badhwar-O'Neill 2014 Galactic Cosmic Ray Flux Model Description, NASA/TP-2015-218569 (2015).

[3] R.A. Nymmik, M.I. Panasyuk, T.I. Pervaya and A.A. Suslov, An analytical model describing dynamics of galactic cosmic ray heavy particles, Adv. Space Res. 14 (1994) 750.

[4] A.J. Tylka, J.H. Adams, P.R. Boberg et al., CREME96: A revision of the Cosmic ray effects on micro-electronics code, IEEE Transactions on Nuclear Science, 44 (1997) 2150.

[5] Space environment (natural and artificial) - galactic cosmic ray model, ISO-15390 (2004).

[6] D. Matthia, T. Berger, A.I. Mrigakshi and G. Reitz, A ready-to-use Galactic Cosmic Ray model, Adv. Space Res. 51 (2013) 329.

[7] N.V. Kuznetsov, E.P. Popova and M.I. Panasyuk, Empirical Model of Long-Time Variations of Galactic Cosmic Ray Particle Fluxes, JGR: Space Physics 122 (2017) 1463.

[8] Y. Shikaze, S. Haino, K. Abe et al., Measurements of 0.2 to $20 \mathrm{GeV} / \mathrm{n}$ cosmic-ray proton and helium spectra from 1997 through 2002 with the BESS spectrometer, Astropart. Phys. 28 (2007) 154.

[9] K. Abe, H. Fuke, S. Haino et al. Measurements of cosmic-ray proton and helium spectra from the BESS-Polar long-duration balloon flights over Antarctica, Astrophys. J. 822 (2016) 65.

[10] J. Alcaraz, B. Alpat, G. Ambrosi et al., Cosmic protons, Physics Letters B, 490, no. 12 (2000) 27.

[11] J. Alcaraz, B. Alpat, G. Ambrosi et al., Helium in near Earth orbit, Physics Letters B 494, no. 34 (2000) 193.

[12] O. Adriani, G.C. Barbarino, G.A. Bazilevskaya et al., Time Dependence of the Proton Flux Measured by PAMELA during the 2006 July-2009 December Solar Minimum, Astrophys. J. $\mathbf{7 6 5}$ (2013) 91.

[13] B.P. Wiebel-Sooth, L. Biermann and H. Meyer, Cosmic rays VII. Individual element spectra: prediction and data, Astronomy and Astrophysics 330 (1998) 389.

[14] J.S. George, K.A. Lave, M.E. Wiedenbeck et al., Elemental composition and energy spectra of galactic cosmic rays during solar cycle 23, Astrophys. J. 698 (2009) 1666.

[15] Z. Fujii and F.B. McDonald, Radial intensity gradients of galactic cosmic rays (1972-1995) in the heliosphere, J. Geophys. Res. 102 (1997), 24201.

[16] F.B. McDonald, Z. Fujii, B. Heikkila, N. Lal and R.E. McGuire, The Radial Distribution of Galactic Cosmic Rays in the Heliosphere at Solar Minimum and Solar Maximum, in proceedings of 28th International Cosmic Ray Conference (2003) 3965. 
[17] P. Kühl, R. Gómez-Herrero and B. Heber, Annual Cosmic Ray Spectra from $250 \mathrm{MeV}$ up to $1.6 \mathrm{GeV}$ from 1995 - 2014. Measured With the Electron Proton Helium Instrument onboard SOHO, Solar Physics 291 (2016) 965.

[18] R.A. Nymmik and A.A. Suslov, Characteristics of Galactic Cosmic Ray Flux Lag Times in the Course of Solar Modulation, Adv. Space Res. 16 (1995) 217.

[19] I.G. Usoskin, K. Mursula and G.A. Kovaltsov, Odd and even cycles in cosmic rays and solar activity, in proceedings of 27th International Cosmic Ray Conference (2001) 3791. 